\title{
Hiperplasia Cutánea Linfoide (Linfocitoma cutis) Secundaria a Fármacos: Hallazgos Clínicos y Morfológicos
}

\author{
Cutaneous Lymphoid Hyperplasia (lymphocytoma cutis) due to Drugs: \\ Clinical and Morphological Findings
}

*,******OScar Tapia E.; ${ }^{* * * * * *}$ Sandra Kam C. \& ***** Yessenia Valenzuela V.

TAPIA, E. O.; KAM, C. S. \& VALENZUELA, V. Y. Hiperplasia cutánea linfoide (linfocitoma cutis) secundaria a fármacos: hallazgos clinicos y morfológicos. Int. J. Morphol., 30(1):170-175, 2012.

RESUMEN: Pseudolinfoma cutáneo es el término utilizado para indicar un grupo de trastornos linfoproliferativos benignos de la piel, siendo el linfocitoma cutis una forma de seudolinfoma de células B. Clínicamente se manifiesta como nódulos o placas solitarias, generalmente localizados en la cara de mujeres jóvenes. Su etiopatogenia estaría relacionada con una respuesta inmunológica local exagerada ante diversos desencadenantes tales como, picadura de insectos, acupuntura, vacunas, tatuajes, medicamentos e infecciones. Se presentan dos casos de pacientes con diagnóstico de linfocitoma cutis realizado en la Unidad de Anatomía Patológica del Hospital Hernán Henríquez de Temuco.

PALABRAS CLAVE: Linfocitoma cutis; Pseudolinfoma; Pseudolinfoma B.

\section{INTRODUCCIÓN}

Pseudolinfoma cutáneo es el término utilizado para indicar un grupo de trastornos linfoproliferativos de la piel, que pueden simular clínica e histológicamente un linfoma cutáneo, correspondiendo por tanto a una proliferación linfoide benigna de estirpe B o T (Dragonetti et al., 2004; Gilliam \& Wood, 2000).

El linfocitoma cutis es uno de los tantos términos utilizados para referirse a un seudolinfoma cutáneo de células B. Clínicamente se manifiesta como nódulos o placas solitarias, de coloración eritematosa o violácea, generalmente localizados en la cara de mujeres jóvenes (Gilliam \& Wood; Rijlaarsdam\& Willemze, 1994; Willemze, 1995; Gambichler et al., 2000; Gissler \& Heininger, 2002).

Esta entidad representaría una respuesta inmunológica local exagerada ante diversos desencadenates tales como, picadura de insectos, acupuntura, vacunas, tatuajes, medicamentos y agentes infecciosos (Gilliam \& Wood; Gambichler et al.; Gissler \& Heininger; Nihal et al., 2003; Madhogaria et al., 2010).
Se presentan dos casos de pacientes con diagnóstico de linfocitoma cutis realizado en la Unidad de Anatomía Patológica del Hospital Hernán Henríquez de Temuco.

\section{REPORTE DE CASOS}

Caso Clínico 1. Mujer de 70 años con hipertensión arterial y diabetes mellitus tipo 2, en tratamiento con losartán, furosemida, glibenclamida y gemfibrozilo.

Consultó a dermatológo por aumento de volumen nodular en zona infrapalpebral izquierda, no pruriginosa, de un año de evolución. El examen clínico constató un nódulo rosado, de superficie lisa y apariencia translúcida de 5 $\mathrm{mm}$ de diámetro mayor, sin otras lesiones asociadas (Fig. 1 A y B). La dermatoscopía evidenció lesión de coloración rosa pálido difuso, sin red pigmentaria que presentaba telangiectasias arboriformes con disposión en corona sin cruzar la lesión (Fig. 1C). Exámenes de laboratorio

* Departamento de Anatomía Patológica. Facultad de Medicina. Universidad de La Frontera. Temuco, Chile.

** Scientific and Technological Bioresource Nucleus (BIOREN).

*** Centro de Genética e Inmunología (CEGIN).

${ }^{* * * * *}$ Residente Dermatología, Pontificia Universidad Católica de Chile, Chile.

${ }^{* * * * * *}$ Dermatóloga, Hospital Pitrufquén, Chile. 
(hemograma, pruebas hepáticas y función renal) resultaron normales.

Se realizó biopsia excisional. El estudio anatomopatológico demostró un denso infiltrado nodular en la dermis superficial y profunda compuesto predominantemente por linfocitos maduros, pleomórficos con tendencia a la formación de folículos linfoides, en partes con centros germinales que presentaban abundantes mitosis normotípicas y cuerpos tingibles. En la zona interfolicular se reconocen linfocitos, plasmocitos, histiocitos y eosinófilos, con preservación de banda de tejido conectivo subepidérmico (zona grenz). No se observó epidermotropismo (Fig. 2 A-B). El estudio inmunohistoquímico demostró positividad para CD20 y CD3, con predominio de linfocitos B; sin restricción de cadenas livianas kapa/lambda. Positividad para Ki67/MIB1 se observó fundamentalmente en los centros germinales, mientras que Bcl-2 en los linfocitos interfoliculares (patrón reactivo) (Fig. 3).

Con estos hallazgos morfológicos e inmunohistoquímicos se diagnosticó una hiperplasia linfoide cutánea (linfocitoma cutis).
Caso Clínico 2. Mujer de 57 años con antecedentes de epilepsia en tratamiento con fenitoína. Derivada a dermatológo por aumento de volumen nodular en surco nasogeniano derecho, de un año de evolución. El examen clínico constató un nódulo rosado, de bordes bien delimitados, superficie lisa y apariencia translúcida de $4 \mathrm{~mm}$ de diámetro mayor; sin otras lesiones asociadas (Fig. 1 D-E). El hemograma y pruebas hepáticas resultaron normales.

Se realizó biopsia excisional, demostrando el estudio anatomo-patológico denso infiltrado nodular en la dermis superficial y profunda compuesto predominantemente por linfocitos maduros, pleomórficos, en partes con formación de centros germinales que presentaban mitosis normotípicas y cuerpos tingibles. En la zona interfolicular se reconocen linfocitos, plasmocitos, histiocitos y eosinófilos. No se observó disposición foliculotrópica ni epidermotropismo, con presencia de zona grenz. El estudio inmunohistoquímico demostró un patrón reactivo con positividad para Bcl-6 y Ki67/MIB-1 fundamentalmente en los centros germinales, mientras que Bcl-2 en los linfocitos interfoliculares; sin restricción de cadenas livianas kapa/ lambda. El infiltrado linfoide mostró positividad para CD20 y CD3, con predominio de linfocitos B.
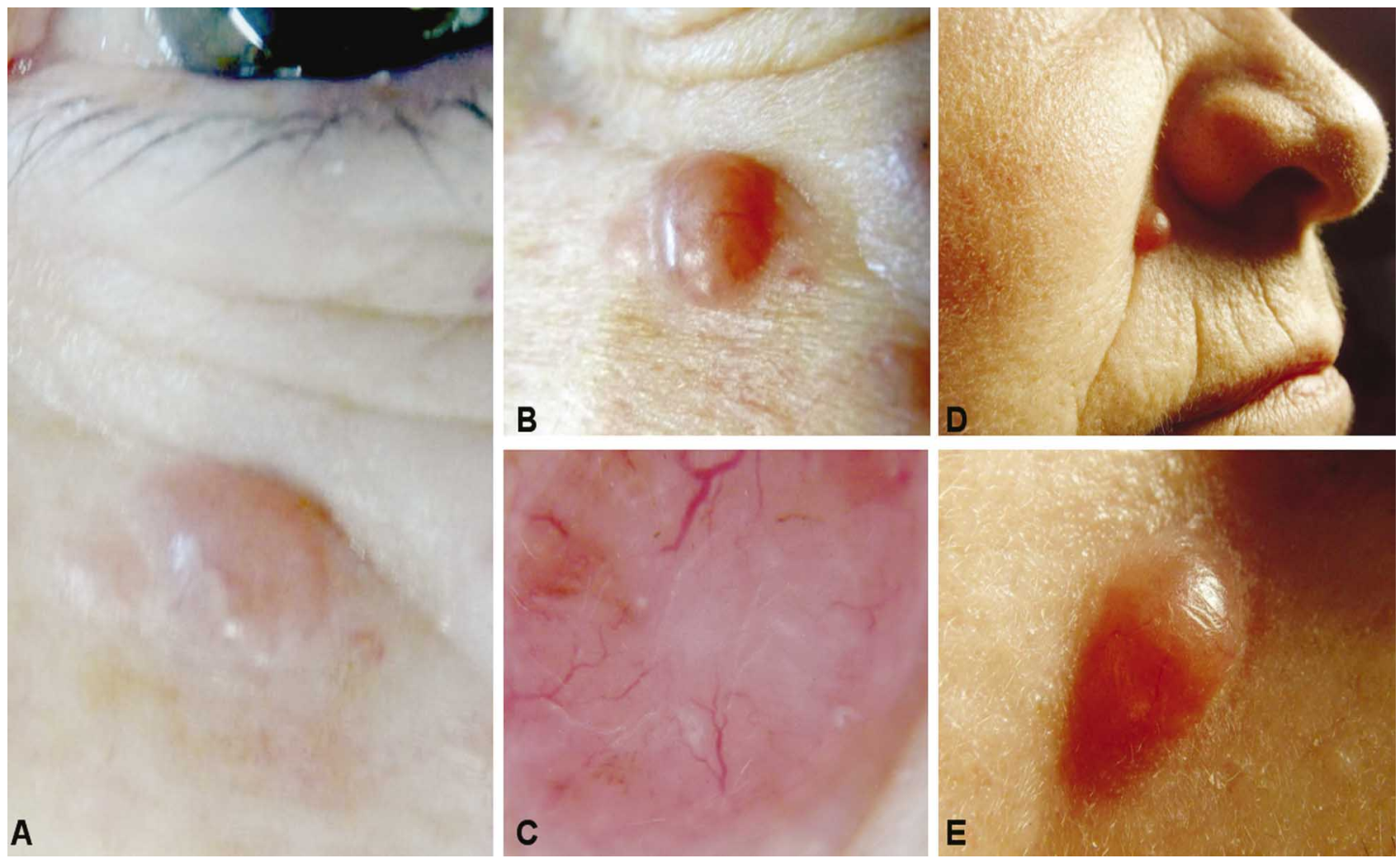

Fig. 1. Ambas pacientes con nódulo solitario de superficie lisa, rosada y apariencia translúcida. Dermatoscopía (C) con lesión de coloración rosa pálido difuso, sin red pigmentaria que presenta telangiectasias arboriformes con disposión en corona sin cruzar la lesión. 

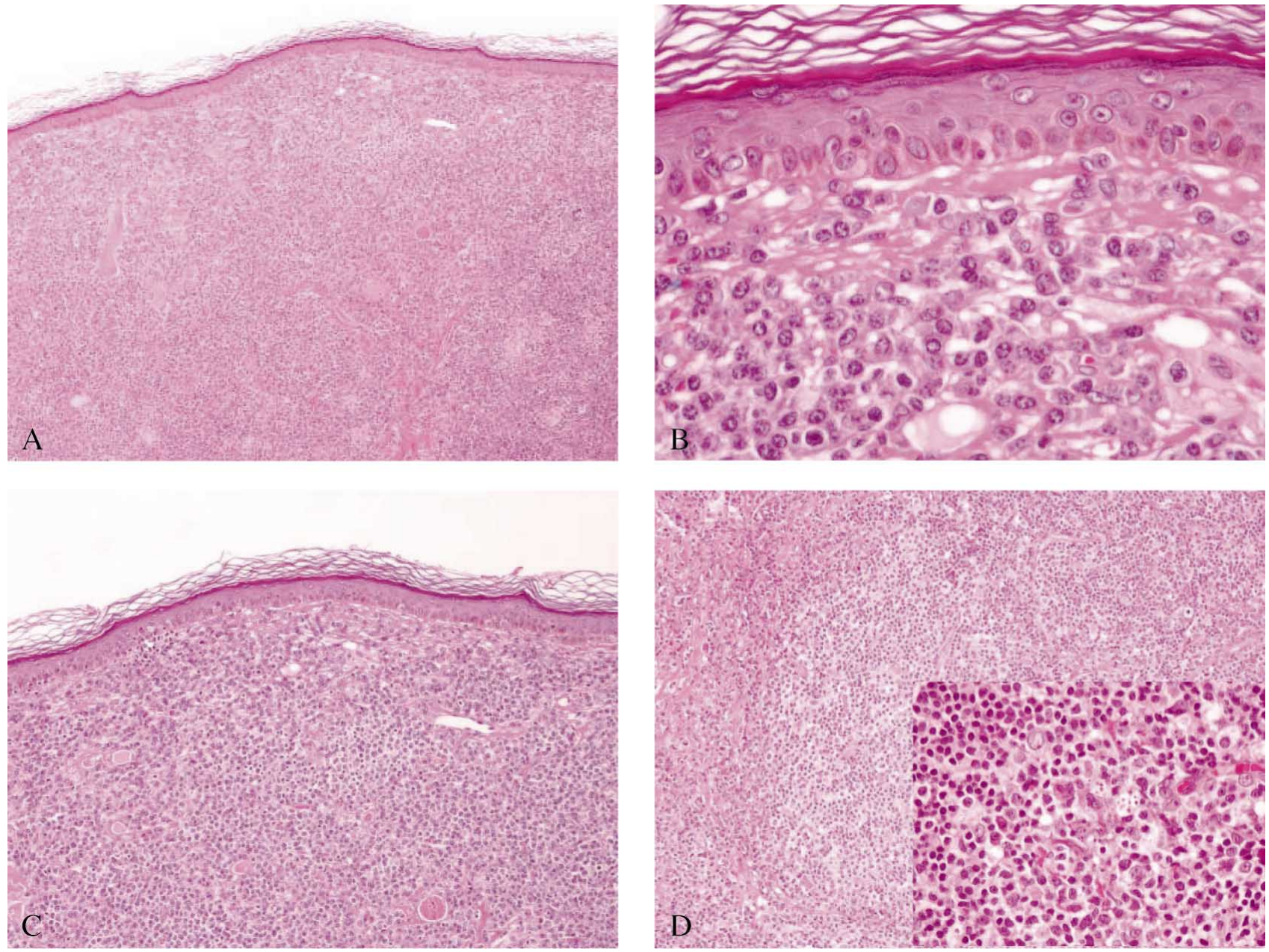

Fig. 2. A (40x, H-E), B (400x, H-E), C (100x H-E), D (100x H-E). A-C: Piel con denso infiltrado nodular linfoide en dermis superficial y profunda en partes con formación de folículos linfoides. No se observa epidermotropismo. D: Folículos linfoides con centros germinales que presentan mitosis normotípicas y cuerpos tingibles.

Los hallazgos morfológicos e inmunohistoquímicos son diagnósticos de hiperplasia linfoide cutánea (Linfocitoma cutis).

\section{DISCUSIÓN}

Los pseudolinfomas cutáneos se clasifican de acuerdo a su población linfoide (linfocitos B o T), patrón de infiltración (nodular o difusa) y los hallazgos clínicos asociados (Carr \& Sanders, 2005).

Mientras que los pseudolinfomas de células T son un grupo heterogeneo de entidades clínico-patológicas que incluyen el reticuloide actínico, dermatitis linfomatoide por contacto, erupción linfomatoide por drogas e infiltrado linfocítico de Jessner, caracterizados histopatológicamente por un denso infiltrado linfocitario en banda en la dermis superior; el pseudolinfoma de células B representa una entidad por si misma, utilizándose diversos términos para referirse a ella: linfocitoma cutis, hiperplasia cutánea linfoide, sarcoide de Spiegler-Fendt, linfadenosis benigna cutis y linfoplasia cutánea (Rijlaarsdam \& Willemze; Willemze; Gambichler et al.).

La etiopatogenia de estas lesiones es desconocida, pudiendo en algunos casos ocurrir como una respuesta inmunológica local reactiva a desencadenates tales como, picadura de insectos, acupuntura, vacunas, tatuajes, fármacos, exposición a alérgenos de contacto o asociado a infecciones por herpes zóster, Borrelia burgdorferi, Leishmania donovani o molusco contagioso. Los fármacos más frecuentemente asociados a estas lesiones son fenitoína, inhibidores de la enzima convertidora de la angiotensina II, antihistamínicos y menos frecuentemente bromocriptina y 

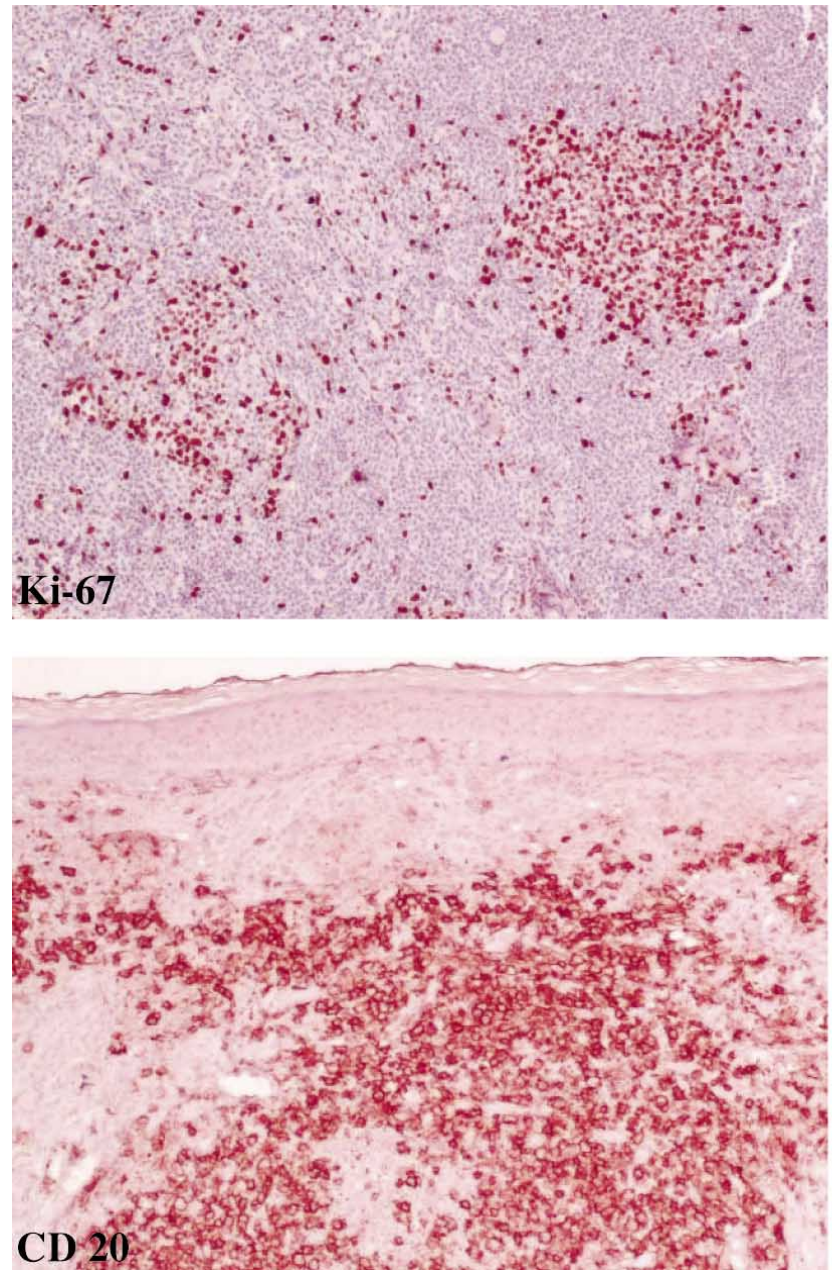
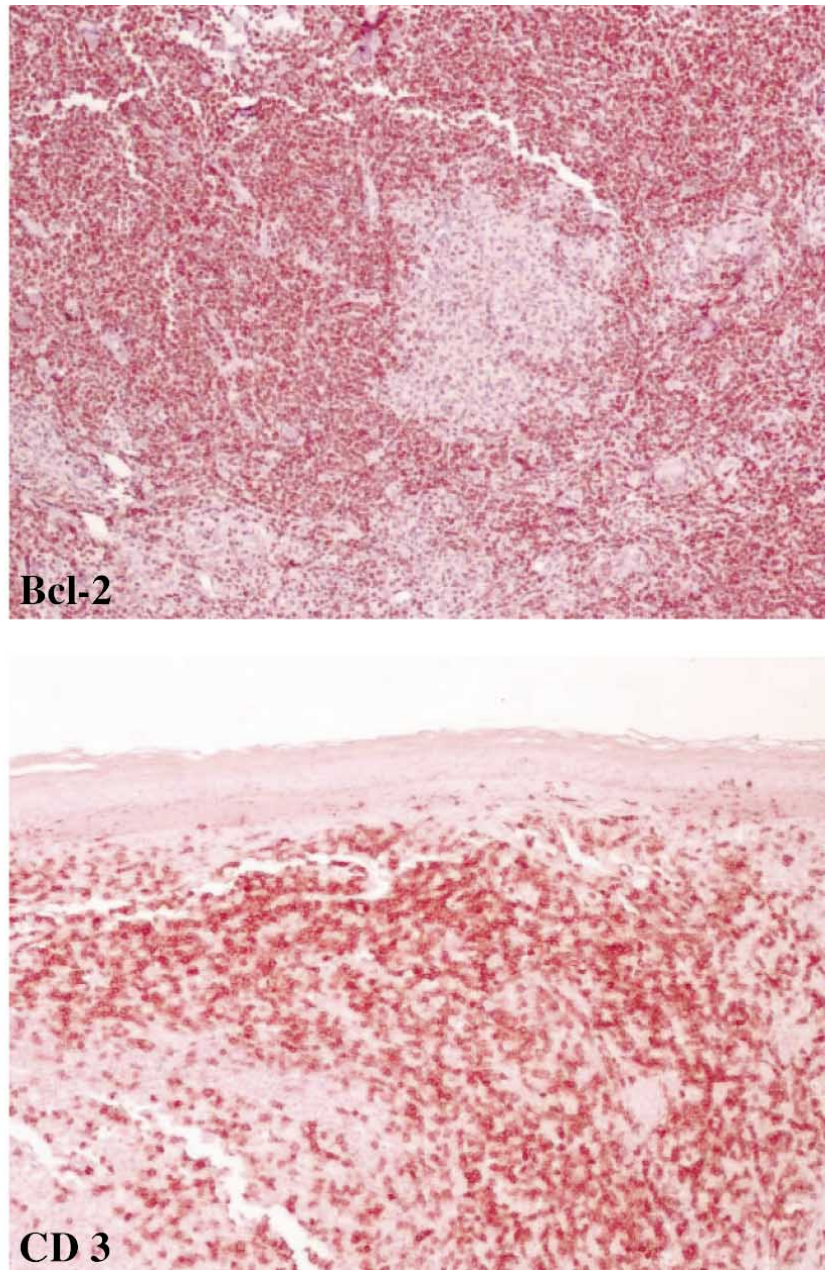

Fig. 3. Inmunohistoquímica con positividad para CD20 y CD3; el Ki67/MIB-1 positivo predominantemente en los centros germinales y Bcl-2 en los linfocitos interfoliculares.

cefuroxima (Gilliam \& Wood; Gambichler et al.; Gissler \& Heininger; Nihal et al.; Madhogaria et al.; Stavrianeas et al., 2007; Magro \& Crowson, 1996; Saeed et al., 2000). En ambas pacientes el uso de losartan (inhibidor de la enzima convertidora de la angiotensina II) y fenitoína son el único factor desencadenante asociado.

El linfocitoma cutis puede presentarse a cualquier edad, predominando en mujeres adultas jóvenes ( $<40$ años), afectando más frecuentemente la cara (mejillas, dorso nasal y lóbulos de los pabellones auriculares); tronco y miembros superiores. Clínicamente se presentan como placas o nódulos solitarios, eritemotosos o violáceos, de hasta $5 \mathrm{~cm}$ de diámetro, de consistencia blanda y superficie lisa, siendo infrecuente la ulceración (Gilliam \& Wood; Rijlaarsdam \& Willemze; Willemze; Gambichler et al.; Gissler \& Heininger). En los dos casos presentados se trató de lesiones únicas, localizadas en la cara, de mujeres de edad mayor.
En el estudio histopatológico se observa habitualmente una epidermis normal, aun cuando puede observarse grados variables de acantosis o hiperplasia seudoepiteliomatosa en caso de picadura de insectos. En la dermis superficial y media se aprecia un denso infiltrado nodular compuesto predominantemente por linfocitos maduros con tendencia a la formación de folículos linfoides, con presencia de mitosis y cuerpos tingibles en los centros germinales. La zona interfolicular está formada por una mezcla de linfocitos, células plasmáticas, histiocitos y eosinófilos, observándose en ocasiones células gigantes multinucleadas. Los vasos sanguíneos en tanto son prominentes, con paredes gruesas y células endoteliales tumefactas. La disposición en "V" del infiltrado linfoide junto con la presencia de una zona grenz entre el infiltrado linfoide y la epidermis y anexos cutáneos constituye un elemento morfológico útil para diferenciarlo de proliferaciones linfoides neoplásicas. Por su parte, el estudio inmunohistoquímico demuestra una proliferación 
linfoide compuesta por una proporción variable de linfocitos B (CD20 y CD79a) y T (CD3 y CD45RO), con predominio $\mathrm{B}$; sin restricción de cadenas livianas kapa/lambda. El índice de proliferación con Ki67/MIB-1 en tanto resulta mayor en los centros germinales a diferencia de los linfomas (Dragonetti et al.; Gilliam \& Wood; Rijlaarsdam \& Willemze; Baldassano et al., 1999; Arai et al., 2005).

El diagnóstico diferencial de esta dermatosis considera cuadros inflamatorios, tumorales benignos y malignos. En este sentido los linfomas cutáneos constituyen el principal diagnóstico diferencial, dado el distinto comportamiento biológico, pronóstico e implicancias terapeúticas; resultando en ocasiones indistinguibles desde el punto de vista clínico e histológico, ya que ambas entidades pueden compartir elementos morfológicos; permitiendo estudios complementarios de inmunohistoquímica y/o biología molecular confirmar el diagnóstico (Dragonetti et al.; Gilliam \& Wood; Gissler \& Heininger; Nihal et al.; Arai et al.).

El tratamiento del linfocitoma cutis considera corticoides sistémicos o intralesionales, criocirugía, cirugía excisional, antibióticos, PUVA terapia, radioterapia y/o inmunosupresores; mientras que en aquellos casos en los que se ha identificado la causa, debe considerarse la suspensión o retiro del agente causal (fármacos, níquel, oro); sin embargo, ninguno de ellos resulta 100\% eficaz (Gilliam \& Wood; Gambichler et al; Stavrianeas et al; Ploysangam et al., 1998). En ambos casos la biopsia excisional resultó terapéutica, sin evidencias de recurrencia luego de 8 y 4 meses de seguimiento.

El curso de esta enfermedad es benigno, desapareciendo las lesiones espontáneamente en semanas o meses, pudiendo, en algunos casos persistir en forma indefinida y/o recidivar; existiendo incluso reportes aislados de progresión a linfoma. Dada la posibilidad de progresión maligna, es que algunos autores plantean la interrogante en cuanto a la naturaleza de estos tumores, postulando que los pseudolinfomas representarían mas bien al espectro benigno en la escala evolutiva hacia un proceso linfoproliferativo maligno (Gilliam \& Wood; Gissler \& Heininger; Madhogaria et al.; Ploysangam et al., 1998; Bouloc et al., 1999).

Finalmente, podemos concluir que el linfocitoma cutis es una proliferación linfoide benigna de células B, localizado generalmente en la cara de mujeres adultas jóvenes; debiendo ser cuidadosamente evaluada desde el punto de vista clínico e histopatológico para descartar una neoplasia linfoide maligna, requiriendo posteriormente estos pacientes control clínico de las lesiones.

TAPIA, E. O.; KAM, C. S. \& VALENZUELA, V. Y. Cutaneous lymphoid hyperplasia (linfocitoma cutis) due to drugs: clinical and morphological findings. Int. J. Morphol., 30(1):170-175, 2012.

SUMMARY: Cutaneous pseudolymphoma is the term used to indicate a group of benign lymphoproliferative disorders of the skin, being the cutaneous lymphomas a form of B-cell pseudolymphoma. Clinically it manifests as solitary nodules or plaques, usually located on the face of young women. Its pathogenesis could be related to an exaggerated local immune response to various causes such as insect bites, acupuncture, vaccinations, tattoos, drugs and infections. We present two cases of patients diagnosed with skin linfocitoma made in the Pathology Unit in Hernán Henríquez Hospital of Temuco.

KEY WORDS: Lymphocytoma cutis; Pseudolymphoma; Pseudolymphoma B.

\section{REFERENCIAS BIBLIOGRÁFICAS}

Arai, E.; Shimizu, M. \& Hirose, T. A review of 55 cases of cutaneous lymphoid hyperplasia: reassessment of the histopathologic findings leading to reclassification of 4 lesions as cutaneous marginal zone lymphoma and 19 as pseudolymphomatous folliculitis. Hum. Pathol., 36:505-11, 2005.

Baldassano, M. F.; Bailey, E. M.; Ferry, J. A.; Harris, N. L. \& Duncan, L. M. Cutaneous lymphoid hyperplasia and cutaneous marginal zone lymphoma: comparison of morphologic and immunophenotypic features. Am. J. Surg. Pathol., 23:88-96, 1999.
Bouloc, A.; Delfau-Larue, M. H.; Lenormand, B.; Meunier, F.; Wechsler, J.; Thomine, E.; Revuz, J.; Farcet, J. P.; Joly, P. \& Bagot, M. Polymerase chain reaction analysis of immunoglobulin gene rearrangement in cutaneous lymphoid hyperplasias. Arch. Dermatol., 135:168-72, 1999.

Carr, R. A. \& Sanders, D. S. A. Inflammatory dermatoses mimicking malignancy (pseudolymphoma). Curr. Diagn. Pathol., 11:245$52,2005$.

Dragonetti, E.; Cianchini, G.; Mastrangelo, L.; Mellone, P. \& Baldi, A. Cutaneus pseudolymphoma: a case report. In Vivo, 18:549-51, 2004. 
Gambichler, T.; Kreuter, J. A.; Stücker, M.; Kobyletzki, G. V.; Altmeyer, P. \& Hoffmann, K. Guess what! Cutaneous lymphoid hyperplasia. Eur. J. Dermatol., 10:635-6, 2000.

Gilliam, A. C. \& Wood, G. S. Cutaneous lymphoid hyperplasias. Semin. Cutan. Med. Surg., 19:133-41, 2000.

Gissler, S. \& Heininger, U. Borrelia lymphocytoma ("lymphadenosis benigna cutis"). Arch. Dis. Child., 87:12, 2002.

Madhogaria, S.; Carr, R. A. \& Gach, J. E. Childhood cutaneous lymphoid hyperplasia following feline scratches. Pediatr. Dermatol., 27:294-7, 2010.

Magro, C. M. \& Crowson, A. N. Drug-induced immune dysregulation as a cause of atypical cutaneous lymphoid infiltrates: a hypothesis. Hum. Pathol., 27:125-32, 1996.

Nihal, M.; Mikkola, D.; Horvath, N.; Gilliam, A. C.; Stevens, S. R.; Spiro, T. P.; Cooper, K. D. \& Wood, G. S. Cutaneous lymphoid hyperplasia: a lymphoproliferative continuum with lymphomatous potential. Hum. Pathol., 34:617-22, 2003.

Ploysangam, T.; Breneman, D. L. \& Mutasim, D. F. Cutaneous pseudolymphoma. J. Am. Acad. Dermatol., 38:877-905, 1998.

Rijlaarsdam, J. U. \& Willemze, R. Cutaneous pseudolymphomas: classification and differential diagnosis. Semin. Dermatol., 13:187-96, 1994.

Saeed, S. A.; Bazza, M.; Zaman, M. \& Ryatt, K. S. Cefuroxime induced lymphomatoid hypersensitivity reaction. Postgrad. Med. J., 76:577-9, 2000.

Stavrianeas, N. G.; Katoulis, A. C.; Bozi, E.; Toumbis-Ioannou, E.; Kanelleas, A. I., Makris, M.; Kalogeromitros, D. \& Panayiotides, I. Cutaneous pseudolymphoma following administration of lornoxicam. Acta Derm. Venereol., 87:453$5,2007$.

Willemze, R. New concepts in the classification of cutaneous lymphomas. Arch. Dermatol., 131:1077-80, 1995.

\author{
Dirección para correspondencia: \\ Dr. Oscar Tapia E. \\ Departamento de Anatomía Patológica \\ Facultad de Medicina \\ Universidad de La Frontera \\ Manuel Montt 112 \\ Código Postal 478-1176 \\ Temuco \\ CHILE
}

Fono: $56-45-296530$

Email: otescalona@gmail.com

Recibido : 24-10-2011

Aceptado: 19-12-2011 